Article

\title{
Generalized Symmetries and mCK Method Analysis of the (2+1)-Dimensional Coupled Burgers Equations
}

\author{
Gangwei Wang ${ }^{1, *,+} \mathbb{D}$, Yixing Liu ${ }^{1,+}$, Shuxin Han ${ }^{1}$, Hua Wang ${ }^{2}$ and Xing Su ${ }^{1}$ \\ 1 School of Mathematics and Statistics, Hebei University of Economics and Business, \\ Shijiazhuang 050061, China \\ 2 Department of Mathematics, Shijiazhuang Tiedao University SiFang College, Shijiazhuang 051132, China \\ * Correspondence: wanggangwei@heuet.edu.cn \\ + These authors contributed equally to this work.
}

Received: 22 October 2019; Accepted: 29 November 2019; Published: 3 December 2019

\begin{abstract}
In this paper, generalized symmetries and mCK method are employed to analyze the (2+1)-dimensional coupled Burgers equations. Firstly, based on the generalized symmetries method, the corresponding symmetries of the $(2+1)$-dimensional coupled Burgers equations are derived. And then, using the mCK method, symmetry transformation group theorem is presented. From symmetry transformation group theorem, a great many of new solutions can be derived. Lastly, Lie algebra for given symmetry group are considered.
\end{abstract}

Keywords: (2+1)-dimensional coupled Burgers equations; generalized symmetries; mCK method; new exact solutions

PACS: 02.20.Sv; 02.30.Jr; 05.45.Yv

MSC: 22E70; 35Qxx

\section{Introduction}

In this paper, by using the generalized symmetries and mCK methods, we will study the following (2+1)-dimensional coupled Burgers equations

$$
\left\{\begin{array}{l}
u_{t}-2 u u_{x}-2 u_{y} v-u_{x x}-u_{y y}=0, \\
v_{t}-2 v v_{y}-2 u v_{x}-v_{x x}-v_{y y}=0 .
\end{array}\right.
$$

It is well known that Burger's types of equations [1,2] can be encountered in many fields, such as fluid mechanics, shock wave formation, boundary layer behavior, continuum traffic simulation, convection dominated diffusion phenomena and so on. As more physical description of the $(2+1)$-dimensional coupled Burgers equations see ([1-11]). There are many authors have been employed various methods to study these types of equations ([1,3-11]). In paper ([8]), one of the authors and his collaborators considered Equation (1) with the Lie symmetry method and suitable Painleve truncated expansions, they derived some exact solutions and conservation laws for Equation (1). In paper ([9]), one of the authors and his collaborators studied a new coupled (2+1)-dimensional Burgers equations, they obtained the symmetry, soliton solutions for the equations. The authors ([12]), based on the Darboux transformation, studied the explicit solutions of a modified 2+1-dimensional coupled Burgers equation. In paper ([13]), they considered finite symmetry transformation group, they also considered localized structures of the (2+1)-dimensional coupled Burgers equation. We generalized their results and give another results. The authors ([14]) give some new exact solutions of Burgers type equations 
with conformable derivative. The authors ([15]) studied time fractional simplified modified Kawahara equation using symmetry method. Motivated by theses papers, it is necessary consider them again in different methods.

This article is a continuation of the previous ones ([8,9]). In order to provide more information about the $(2+1)$ dimensional Burgers equation, it is necessary to consider the coupled $(2+1)$ dimensional Burgers equation in different ways. Perhaps in this way, we can find the connections and differences between the different methods.

Our aim in this work is to consider the (2+1)-dimensional coupled Burgers equations using the generalized symmetries method and mCK method. This article is divided into the following parts: Firstly, we consider the (2+1)-dimensional coupled Burgers equations using the generalized symmetry method. Secondly, mCK method is employed to derive new exact solutions for the (2+1)-dimensional coupled Burgers equations. Lie algebra for given symmetry group also derived. Lastly, the conclusions are presented.

\section{Generalized Symmetries for the (2+1)-Dimensional Coupled Burgers Equations}

Now we studied the symmetries of Equation (1.1) by using the generalized symmetry method ([16-18]).

If nonlinear evolution equation are given, that is to say,

$$
F\left(t, x, y, u, u_{t}, u_{x}, u_{y}, v, v_{t} \cdots\right)=0
$$

for all solutions of $u$, we say a function $\sigma$ is a symmetry of Equation (2), if it satisfies the following equation

$$
F^{\prime}(u, v) \sigma=0
$$

where

$$
F^{\prime}(u, v) \sigma=\frac{\partial F}{\partial u} \sigma+\frac{\partial F}{\partial u_{t}} \sigma_{t}+\frac{\partial F}{\partial u_{x}} \sigma_{x}+\frac{\partial F}{\partial v_{t}} \sigma_{t}+\frac{\partial F}{\partial v_{x}} \sigma_{x}+\frac{\partial F}{\partial u_{y}} \sigma_{y}+\frac{\partial F}{\partial u_{t x}} \sigma_{t x}+\frac{\partial F}{\partial u_{x x}} \sigma_{x x}+\cdots
$$

It is immediately generate the symmetry equations of Equation (1) as follows

$$
\begin{array}{r}
\sigma_{1 t}-2 \sigma_{1} u_{x}-2 u \sigma_{1 x}-2 \sigma_{1 y} v-2 u_{y} \theta-\sigma_{1 x x}-\sigma_{1 y y}=0, \\
\theta_{t}-2 \theta_{x} u-2 \sigma_{1} v_{x}-2 v_{y} \theta-2 \theta_{y} v-\theta_{x x}-\theta_{y y}=0 .
\end{array}
$$

In order to get our goal, we set the following functions,

$$
\begin{gathered}
\sigma_{1}=a u_{x}+b u_{t}+c u_{y}+e u+g v+l, \\
\theta=a v_{x}+b v_{t}+c v_{y}+m v+h u+k,
\end{gathered}
$$

where $a, b, c, e, g, l, m, h$ and $k$ are functions of $t, x$ and $y$ to be determined.

Substituting (6) into (5) and using (1), and replace $u_{t}$ by $2 u u_{x}+2 u_{y} v+u_{x x}+u_{y y}$, and $v_{t}$ by $2 v v_{y}+2 u v_{x}+v_{x x}+v_{y y}$. And then we get the determining equations, it is generate the following results

$$
\begin{aligned}
& a=\left(c_{1} t+c_{2}\right) x+2 c_{6} t-c_{3} y+c_{7}, \quad b=c_{1} t^{2}+2 c_{2} t+c_{4}, \\
& c=\left(c_{1} t+c_{2}\right) y+2 c_{5} t+c_{3} x+c_{8}, e=m=c_{1} t+c_{2}, g=c_{3}, \\
& l=\frac{c_{1}}{2} x+c_{6}, h=-c_{3}, k=\frac{c_{1}}{2} y+c_{5},
\end{aligned}
$$

here $c_{i}(i=1,2 \cdots 8)$ are arbitrary constants. Putting (7) into (6), we have 


$$
\begin{aligned}
& \sigma_{1}=\left(\left(c_{1} t+c_{2}\right) x+2 c_{6} t-c_{3} y+c_{7}\right) u_{x}+\left(c_{1} t^{2}+2 c_{2} t+c_{4}\right) u_{t}+\left(\left(c_{1} t\right.\right. \\
& \left.\left.\quad+c_{2}\right) y+2 c_{5} t+c_{3} x+c_{8}\right) u_{y}+\left(c_{1} t+c_{2}\right) u+c_{3} v+\frac{c_{1}}{2} x+c_{6}, \\
& \theta=\left(\left(c_{1} t+c_{2}\right) x+2 c_{6} t-c_{3} y+c_{7}\right) u_{x}+\left(c_{1} t^{2}+2 c_{2} t+c_{4}\right) u_{t}+\left(\left(c_{1} t\right.\right. \\
& \left.\left.\quad+c_{2}\right) y+2 c_{5} t+c_{3} x+c_{8}\right) u_{y}+\left(c_{1} t+c_{2}\right) v-c_{3} u+\frac{c_{1}}{2} y+c_{5} .
\end{aligned}
$$

In other words, we get

$$
\begin{aligned}
\sigma & =c_{1}\left[x t u_{x}+t^{2} u_{t}+t y u_{y}+\left(t u+\frac{1}{2} x\right) u+\left(t v+\frac{1}{2} y\right) v\right] \\
& +c_{2}\left[x u_{x}+2 t u_{t}+y u_{y}+u+v\right]+c_{3}\left[-y u_{x}+x u_{y}+v-u\right] \\
& +c_{4} u_{t}+c_{5}\left(2 t u_{y}+1\right)+c_{6}\left(2 t u_{x}+1\right)+c_{7} u_{x}+c_{8} u_{y} .
\end{aligned}
$$

So we derived the equivalent vector expression is follows

$$
\begin{aligned}
V & =\left(\left(c_{1} t+c_{2}\right) x+2 c_{6} t-c_{3} y+c_{7}\right) \frac{\partial}{\partial x}+\left(c_{1} t^{2}+2 c_{2} t+c_{4}\right) \frac{\partial}{\partial t}+\left(\left(c_{1} t+c_{2}\right) y+2 c_{5} t+c_{3} x+c_{8}\right) \frac{\partial}{\partial y} \\
& -\left(\left(c_{1} t+c_{2}\right) u+c_{3} v+\frac{c_{1}}{2} x+c_{6}\right) \frac{\partial}{\partial u}-\left(\left(c_{1} t+c_{2}\right) v-c_{3} u+\frac{c_{1}}{2} y+c_{5}\right) \frac{\partial}{\partial v} .
\end{aligned}
$$

In fact, it is coincide with the obtained vector field $V_{i}(i=1,2 \cdots 8)$ of paper [8]. That is to say, for this assumption, it is the same results for the Lie symmetry method. However, for other assumption, we do not know what happened, we will studied them in future work. In paper [3], the authors derived the similarity reductions in detail. Here, we do not list all of them.

\section{Non-Lie Symmetry Groups for the (2+1)-Dimensional Coupled Burgers Equations}

In this section, we use the mCK method [19] to analyze the (2+1)-dimensional coupled Burgers equations. Lou et al. proposed a simple method (called the mCK method) to get the non-Lie point symmetry group [19] of the given nonlinear evolution equations. Firstly, we consider the following transformation

$$
\begin{aligned}
& u(x, y, t)=\alpha_{1}+\beta_{1} U(\xi, \eta, \tau)+\gamma_{1} V(\xi, \eta, \tau), \\
& v(x, y, t)=\alpha_{2}+\beta_{2} V(\xi, \eta, \tau)+\gamma_{2} U(\xi, \eta, \tau),
\end{aligned}
$$

where $\alpha_{1}=\alpha_{1}(x, y, t), \beta_{1}=\beta_{1}(x, y, t), \alpha_{2}=\alpha_{2}(x, y, t), \beta_{2}=\beta_{2}(x, y, t), \gamma_{1}=\gamma_{1}(x, y, t), \gamma_{2}=$ $\gamma_{2}(x, y, t), \xi=\xi(x, y, t), \eta=\eta(x, y, t)$ and $\tau=\tau(x, y, t)$ are functions of $\{x, y, t\}$ to be further determined. In this case, based on the mCK method, it is requiring $U(\xi, \eta, \tau)$ and $V(\xi, \eta, \tau)$ are satisfy the same as $u(x, y, t)$ and $v(x, y, t)$ under the transformation $\{u, v, x, y, t\} \rightarrow\{U, V, \xi, \eta, \tau\}$.

Putting Equation (11) into Equation (1) and requiring that $U(\xi, \eta, \tau)$ and $V(\xi, \eta, \tau)$ satisfy the same equations as Equation (1)

$$
\left\{\begin{array}{l}
U_{\tau}-2 U U_{\xi}-2 U_{\eta} V-U_{\xi \xi}-U_{\eta \eta}=0, \\
V_{\tau}-2 V V_{\eta}-2 V_{\xi} U-V_{\xi \xi}-V_{\eta \eta}=0 .
\end{array}\right.
$$

Replacing $U_{\tau}$ by $2 U U_{\xi}+2 U_{\eta} V+U_{\xi \xi}+U_{\eta \eta}$, and $V_{\tau}$ by $2 V V_{\eta}+2 V_{\xi} U+V_{\xi \xi}+V_{\eta \eta}$, we can arrive at complicated differential equations with $U, V$ and their different derivatives. After that, we ruling out the coefficients of $U, V$ and their derivatives, one obtains over-determined equations for the unknown functions of $\left\{\alpha_{1}, \beta_{1}, \alpha_{2}, \beta_{2}, \gamma_{1}, \gamma_{2}, \xi, \eta, \tau\right\}$. 


$$
\begin{aligned}
& \tau_{x}=0, \tau_{y}=0, \\
& \gamma_{1 t}-\gamma_{1 x x}-\gamma_{1 y y}-2 \alpha_{1} \gamma_{1 x}-2 \gamma_{1} \alpha_{1 x}-2 \alpha_{2} \gamma_{1 y}-2 \beta_{2} \alpha_{1 y}=0 \text {, } \\
& \beta_{1 t}-\beta_{1 x x}-\beta_{1 y y}-2 \alpha_{1} \beta_{1 x}-2 \beta_{1} \alpha_{1 x}-2 \alpha_{2} \beta_{1 y}-2 \gamma_{2} \alpha_{1 y}=0, \\
& \alpha_{1 t}-2 \alpha_{1} \alpha_{1 x}-2 \alpha_{2} \alpha_{1 y}-\alpha_{1 x x}-\alpha_{1 y y}=0 \text {, } \\
& \beta_{1}\left(\tau_{t}-\xi_{x}^{2}-\xi_{y}^{2}\right)=0, \gamma_{1}\left(\tau_{t}-\xi_{x}^{2}-\xi_{y}^{2}\right)=0, \\
& \beta_{1} \xi_{t}-\beta_{1} \xi_{y y}-\beta_{1} \xi_{x x}-2 \beta_{1 x} \xi_{x}-2 \beta_{1 y} \xi_{y}-2 \alpha_{1} \beta_{1} \xi_{x}-2 \alpha_{2} \beta_{1} \xi_{y}=0 \text {, } \\
& \gamma_{1} \xi_{t}-\gamma_{1} \xi_{y y}-\gamma_{1} \xi_{x x}-2 \gamma_{1 x} \xi_{x}-2 \gamma_{1 y} \xi_{y}-2 \alpha_{1} \gamma_{1} \xi_{x}-2 \alpha_{2} \gamma_{1} \xi_{y}=0 \text {, } \\
& \beta_{1}\left(\tau_{t}-\eta_{x}^{2}-\eta_{y}^{2}\right)=0, \gamma_{1}\left(\tau_{t}-\eta_{x}^{2}-\eta_{y}^{2}\right)=0 \text {, } \\
& \beta_{1} \eta_{t}-\beta_{1} \eta_{y y}-\beta_{1} \eta_{x x}-2 \beta_{1 x} \eta_{x}-2 \beta_{1 y} \eta_{y}-2 \alpha_{1} \beta_{1} \eta_{x}-2 \alpha_{2} \beta_{1} \eta_{y}=0 \text {, } \\
& \gamma_{1} \eta_{t}-\gamma_{1} \eta_{y y}-\gamma_{1} \eta_{x x}-2 \gamma_{1 x} \eta_{x}-2 \gamma_{1 y} \eta_{y}-2 \alpha_{1} \gamma_{1} \eta_{x}-2 \alpha_{2} \gamma_{1} \eta_{y}=0 \text {, } \\
& -2 \beta_{1 y} \gamma_{2}-2 \beta_{1 x} \beta_{1}=0,-2 \gamma_{1 x} \gamma_{1}-2 \gamma_{1 y} \beta_{2}=0 \text {, } \\
& 2 \gamma_{1}\left(\tau_{t}-\gamma_{2} \xi_{y}-\beta_{1} \xi_{x}\right)=0,2 \beta_{1}\left(\tau_{t}-\beta_{2} \eta_{y}-\gamma_{1} \eta_{x}\right)=0, \\
& -2 \beta_{1} \gamma_{1} \eta_{x}-2 \gamma_{1} \gamma_{2} \eta_{y}=0,-2 \beta_{1} \gamma_{1} \xi_{x}-2 \beta_{1} \beta_{2} \xi_{y}=0, \\
& 2 \beta_{1}\left(\tau_{t}-\beta_{1} \xi_{x}-\gamma_{2} \xi_{y}\right)=0,2 \gamma_{1}\left(\tau_{t}-\gamma_{1} \eta_{x}-\beta_{2} \eta_{y}\right)=0 . \\
& -2 \beta_{1}\left(\gamma_{2} \eta_{y}+\beta_{1} \eta_{x}\right)=0,-2 \gamma_{1}\left(\gamma_{1} \xi_{x}+\beta_{2} \xi_{y}\right)=0, \\
& -2 \gamma_{1}\left(\eta_{x} \xi_{x}+\eta_{y} \xi_{y}\right)=0,-2 \beta_{1}\left(\eta_{x} \xi_{x}+\eta_{y} \xi_{y}\right)=0, \\
& -2 \beta_{1} \gamma_{1 x}-2 \gamma_{1} \beta_{1 x}-2 \gamma_{1 y} \gamma_{2}-2 \beta_{1 y} \beta_{2}=0 \text {, } \\
& \alpha_{2 t}-2 \alpha_{1} \alpha_{2 x}-2 \alpha_{2} \alpha_{2 y}-\alpha_{2 x x}-\alpha_{2 y y}=0 \text {, } \\
& \gamma_{2} \eta_{t}-\gamma_{2} \eta_{y y}-\gamma_{2} \eta_{x x}-2 \gamma_{2 x} \eta_{x}-2 \gamma_{2 y} \eta_{y}-2 \alpha_{1} \gamma_{2} \eta_{x}-2 \alpha_{2} \gamma_{2} \eta_{y}=0 \text {, } \\
& \gamma_{2} \xi_{t}-\gamma_{2} \xi_{y y}-\gamma_{2} \xi_{x x}-2 \gamma_{2 x} \xi_{x}-2 \gamma_{2 y} \xi_{y}-2 \alpha_{1} \gamma_{2} \xi_{x}-2 \alpha_{2} \gamma_{2} \xi_{y}=0 \text {, } \\
& \beta_{2} \xi_{t}-\beta_{2} \xi_{y y}-\beta_{2} \xi_{x x}-2 \beta_{2 x} \xi_{x}-2 \beta_{2 y} \xi_{y}-2 \alpha_{1} \beta_{2} \xi_{x}-2 \alpha_{2} \beta_{2} \xi_{y}=0 \text {, } \\
& \beta_{2} \eta_{t}-\beta_{2} \eta_{y y}-\beta_{2} \eta_{x x}-2 \beta_{2 x} \eta_{x}-2 \beta_{2 y} \eta_{y}-2 \alpha_{1} \beta_{2} \eta_{x}-2 \alpha_{2} \beta_{2} \eta_{y}=0 \text {, } \\
& \beta_{2}\left(\tau_{t}-\eta_{x}^{2}-\eta_{y}^{2}\right)=0, \gamma_{2}\left(\tau_{t}-\eta_{x}^{2}-\eta_{y}^{2}\right)=0 \text {, } \\
& \beta_{2}\left(\tau_{t}-\xi_{x}^{2}-\xi_{y}^{2}\right)=0, \gamma_{2}\left(\tau_{t}-\xi_{x}^{2}-\xi_{y}^{2}\right)=0 \text {, } \\
& \beta_{2 t}-\beta_{2 x x}-\beta_{2 y y}-2 \alpha_{1} \beta_{2 x}-2 \beta_{2} \alpha_{2 y}-2 \alpha_{2} \beta_{2 y}-2 \gamma_{1} \alpha_{2 x}=0 \text {, } \\
& \gamma_{2 t}-\gamma_{2 x x}-\gamma_{2 y y}-2 \alpha_{1} \gamma_{2 x}-2 \gamma_{2} \alpha_{2 y}-2 \alpha_{2} \gamma_{2 y}-2 \beta_{1} \alpha_{2 x}=0 \text {, } \\
& -2 \beta_{2 y} \beta_{2}-2 \beta_{2 x} \gamma_{1}=0,-2 \gamma_{2 x} \beta_{1}-2 \gamma_{2 y} \gamma_{2}=0, \\
& 2 \beta_{2}\left(\tau_{t}-\gamma_{2} \xi_{y}-\beta_{1} \xi_{x}\right)=0,2 \gamma_{2}\left(\tau_{t}-\beta_{2} \eta_{y}-\gamma_{1} \eta_{x}\right)=0, \\
& -2 \beta_{1} \beta_{2} \eta_{x}-2 \beta_{2} \gamma_{2} \eta_{y}=0,-2 \gamma_{2} \gamma_{1} \xi_{x}-2 \beta_{2} \gamma_{2} \xi_{y}=0 \text {, } \\
& 2 \gamma_{2}\left(\tau_{t}-\beta_{1} \xi_{x}-\gamma_{2} \xi_{y}\right)=0,2 \beta_{2}\left(\tau_{t}-\gamma_{1} \eta_{x}-\beta_{2} \eta_{y}\right)=0, \\
& -2 \gamma_{2}\left(\gamma_{2} \eta_{y}+\beta_{1} \eta_{x}\right)=0,-2 \beta_{2}\left(\gamma_{1} \xi_{x}+\beta_{2} \xi_{y}\right)=0, \\
& -2 \gamma_{2}\left(\eta_{x} \xi_{x}+\eta_{y} \xi_{y}\right)=0,-2 \beta_{2}\left(\eta_{x} \xi_{x}+\eta_{y} \xi_{y}\right)=0, \\
& -2 \beta_{2} \gamma_{2 y}-2 \gamma_{2} \beta_{2 y}-2 \gamma_{2 x} \gamma_{1}-2 \beta_{1 x} \beta_{1}=0 \text {. }
\end{aligned}
$$

It is clear that it is rather difficult to solve the over determined equations. So we consider only $\gamma_{1}=\gamma_{2}=0$ on these equations. Then solving the above equations, we get

$$
\tau=\tau(t), \xi=\tau_{t}^{\frac{1}{2}} x+b(t), \eta=\tau_{t}^{\frac{1}{2}} y+c(t), \beta_{1}=\tau_{t}^{\frac{1}{2}}, \alpha_{1}=\frac{\frac{1}{2} \tau_{t} x+b_{t} \tau_{t}^{\frac{1}{2}}}{2 \tau_{t}}, \beta_{2}=\tau_{t}^{\frac{1}{2}}, \alpha_{2}=\frac{\frac{1}{2} \tau_{t} y+c_{t} \tau_{t}^{\frac{1}{2}}}{2 \tau_{t}},
$$

where $b(t)$ and $c(t)$ are arbitrary functions. The results of paper ([13]) is the special cases for our results. 
On the other side, if we let $\beta_{1}=\beta_{2}=0$, after some calculations, we get

$$
\tau=\tau(t), \xi=\tau_{t}^{\frac{1}{2}} y+b(t), \eta=\tau_{t}^{\frac{1}{2}} x+c(t), \gamma_{1}=\tau_{t}^{\frac{1}{2}}, \alpha_{1}=\frac{\frac{1}{2} \tau_{t} x+b_{t} \tau_{t}^{\frac{1}{2}}}{2 \tau_{t}}, \gamma_{2}=\tau_{t}^{\frac{1}{2}}, \alpha_{2}=\frac{\frac{1}{2} \tau_{t} y+c_{t} \tau_{t}^{\frac{1}{2}}}{2 \tau_{t}},
$$

where $b(t)$ and $c(t)$ are arbitrary functions. It is easy to see that only $\xi$ and $\eta$ is different.

In summary, based on the above analysis, for the $(2+1)$-dimensional coupled Burgers equations, the following symmetry transformation group theorem are obtained:

Theorem 1. If $\{U=U(\xi, \eta, \tau), V=V(\xi, \eta, \tau)\}$ are solutions of Equation (1), then the solutions of Equation (1) $u, v$ can be expressed by

$$
u=\frac{\frac{1}{2} \tau_{t t} x+b_{t} \tau_{t}^{\frac{1}{2}}}{2 \tau_{t}}+\tau_{t}^{\frac{1}{2}} U(\xi, \eta, \tau), v=\frac{\frac{1}{2} \tau_{t t} y+c_{t} \tau_{t}^{\frac{1}{2}}}{2 \tau_{t}}+\tau_{t}^{\frac{1}{2}} V(\xi, \eta, \tau),
$$

where $\xi, \eta, \tau, b(t)$ and $c(t)$ are decided by Equation (17) or Equation (18).

In this case, based on Theorem 1, we can derive abundant of new solutions of the (2+1)-dimensional coupled Burgers equations from its given solutions, for example, we select the following the single kink solutions [6] as seed solution.

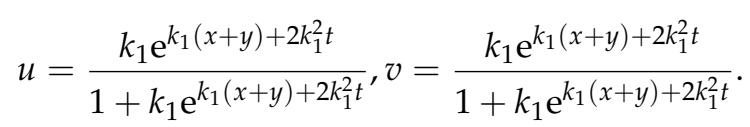

From Theorem 1, one can present new solutions of Equation (1) as follows

$$
u=\frac{\frac{1}{2} \tau_{t t} x+b_{t} \tau_{t}^{\frac{1}{2}}}{2 \tau_{t}}+\tau_{t}^{\frac{1}{2}} \frac{k_{1} \mathrm{e}^{k_{1}(\xi+\eta)+2 k_{1}^{2} \tau}}{1+k_{1} \mathrm{e}^{k_{1}(\xi+\eta)+2 k_{1}^{2} \tau}}, v=\frac{\frac{1}{2} \tau_{t t} y+c_{t} \tau_{t}^{\frac{1}{2}}}{2 \tau_{t}}+\tau_{t}^{\frac{1}{2}} \frac{k_{1} \mathrm{e}^{k_{1}(\xi+\eta)+2 k_{1}^{2} \tau}}{1+k_{1} \mathrm{e}^{k_{1}(\xi+\eta)+2 k_{1}^{2} \tau}},
$$

where $\xi, \eta, \tau, b(t)$ and $c(t)$ are decided by Equation (17) or Equation (18). When taking $\tau=t, b(t)=c(t)=0$, the solutions determined by Equation (21) are the results in Ref. [6]. Thus, it is clear that, we generalize the corresponding results in Ref. [6].

Remark 1. Following the same process, a great many of new solutions can be derived through given solutions [3,6,8] for the coupled Burgers equations. Thus we generalize the corresponding results in $[3,6,8]$.

\section{Lie Algebra for Given Symmetry Group}

Now, from Theorem 1, it can be found that the Lie point symmetry group, in fact, is a special case of the given symmetry group. If we let [13]

$$
\tau(t)=t+\varepsilon f(t), b(t)=\varepsilon h(t), c(t)=\varepsilon m(t),
$$

where $\varepsilon$ is an infinitesimal parameter, $f, h$ and $m$ are arbitrary functions of $t$.

We can derive the Lie point symmetries

$$
\begin{aligned}
& \sigma_{1}=\left(\frac{1}{2} x f_{t}+h(t)\right) u_{x}+\left(\frac{1}{2} y f_{t}+m(t)\right) u_{y}+f(t) u_{t}+\left(\frac{1}{4} x f_{t t}+\frac{1}{2} h_{t}+\frac{1}{2} u f_{t}\right) \\
& \sigma_{2}=\left(\frac{1}{2} x f_{t}+h(t)\right) u_{x}+\left(\frac{1}{2} y f_{t}+m(t)\right) u_{y}+f(t) u_{t}+\left(\frac{1}{4} y f_{t t}+\frac{1}{2} m_{t}+\frac{1}{2} v f_{t}\right) .
\end{aligned}
$$


The corresponding equivalent vectors expression as follows

$$
\begin{aligned}
V & =\left(\frac{1}{2} x f_{t}+h(t)\right) \frac{\partial}{\partial x}+f(t) \frac{\partial}{\partial t}+\left(\frac{1}{2} y f_{t}+m(t)\right) \frac{\partial}{\partial y} \\
& -\left(\frac{1}{4} x f_{t t}+\frac{1}{2} h_{t}+\frac{1}{2} u f_{t}\right) \frac{\partial}{\partial u}-\left(\frac{1}{4} y f_{t t}+\frac{1}{2} m_{t}+\frac{1}{2} v f_{t}\right) \frac{\partial}{\partial v} .
\end{aligned}
$$

As the functions $f, g$ and $m$ are arbitrary, thus the corresponding Lie algebra is an infinite-dimensional Lie algebra.

If we set $f=2 c_{1} t+c_{2}, m=2 c_{3} t+c_{4}, h=2 c_{5} t+c_{6}$, one obtains

$$
V=\left(c_{1} x+2 c_{5} t+c_{6}\right) \frac{\partial}{\partial x}+\left(2 c_{1} t+c_{2}\right) \frac{\partial}{\partial t}+\left(c_{1} y+2 c_{3} t+c_{4}\right) \frac{\partial}{\partial y}-\left(c_{5}+c_{1} u\right) \frac{\partial}{\partial u}-\left(c_{3}+c_{1} v\right) \frac{\partial}{\partial v} .
$$

From (25), we can derive the corresponding Lie algebra is spanned by the following six vector fields

$$
\begin{aligned}
& V_{1}=\frac{\partial}{\partial x}, V_{2}=\frac{\partial}{\partial y}, V_{3}=\frac{\partial}{\partial t}, V_{4}=2 t \frac{\partial}{\partial x}-\frac{\partial}{\partial u}, \\
& V_{5}=2 t \frac{\partial}{\partial y}-\frac{\partial}{\partial v}, V_{6}=x \frac{\partial}{\partial x}+2 t \frac{\partial}{\partial t}+y \frac{\partial}{\partial y}-u \frac{\partial}{\partial u}-v \frac{\partial}{\partial v} .
\end{aligned}
$$

It is clear that the symmetry generators form a closed Lie algebra.

\section{Conclusions}

In this letter, according to the generalized symmetries analysis method, we find out that the corresponding symmetries. In fact, for our assumption $\left(\sigma_{1}=a u_{x}+b u_{t}+c u_{y}+e u+g v+l, \theta=\right.$ $a v_{x}+b v_{t}+c v_{y}+m v+h u+k$,), that is to say, they are linear functions, it is coincided with the classical Lie symmetry method. However, for other setting, if they are nonlinear functions, what will be happened? It is worth studying them in future works. Meanwhile, based on the mCK method, we also give the non-Lie symmetry groups for the (2+1)-dimensional coupled Burgers equations, which have important application in many fields. These results are helpful for explaining complex physical phenomena. Here, we only considered the special cases, it is also need to be investigated them for the general cases. Until now, the classical symmetry, the local conservation laws are presented. There are many issues need to be considered, such as, nonlocal symmetries, and (2+1)-dimensional coupled Burgers equations with variable coefficients as well as their discrete versions, and so on.

Data Availability: The data used to support the findings of this study are included within the article.

Author Contributions: These authors contributed equally to this work.

Funding: The paper is supported by Natural Science Foundation of Hebei Province of China (No. A2018207030) and Youth Key Program of Hebei University of Economics and Business (2018QZ07), NNSF of China (No. 11801133, 11801132).

Conflicts of Interest: The authors declare that they have no conflict of interest.

\section{References}

1. Burgers, J.M. The Nonlinear Diffusion Equation; Reiedl: Dordtrecht, The Netherlands, 1974.

2. Whitham G.B. Linear and Nonlinear Waves, 1st ed.; John Wiley-Interscience: New York, NY, USA, 1999.

3. Tamizhmani, K.M.; Punithavathi, P. Similarity reductions and Painlev'e property of the coupled higher-dimensional Burgers' equation. Int. J. Nonlin. Mech. 1991, 26, 427-438. [CrossRef]

4. Wazwaz, A.M. Multiple-front solutions for the Burgers equation and the coupled Burgers equations. Appl. Math. Comput. 2007, 190, 1198-1206. [CrossRef]

5. Wazwaz, A.M. Multiple kink solutions for M-component Burgers equations in (1+1)-dimensions and (2+1)-dimensions. Appl. Math. Comput. 2010, 217, 3564-3570. [CrossRef] 
6. Wazwaz, A.M. Multiple kink solutions and multiple singular kink solutions for the (2+1)-dimensional Burgers equations. Appl. Math. Comput. 2008, 204, 817-823. [CrossRef]

7. Wang, Q.; Song, L.N.; Zhang, H.Q. A new coupled sub-equations expansion method and novel complexiton solutions of (2+1)-dimensional Burgers equation. Appl. Math. Comput. 2007, 186, 632-637. [CrossRef]

8. Wang, G.; Xu, T.; Biswas, A. Topological solitons and conservation laws of the coupled Burgers equations. Romanian Rep. Phys. 2014, 66, 274-285.

9. Wang, G.; Fakhar, K.; Kara, A.H. Soliton solutions and group analysis of a new coupled $(2+1)$-dimensional Burgers equations. Acta Phys. Pol. B 2015, 46, 923-930. [CrossRef]

10. Ma, W.X. A hierarchy of coupled Burgers systems possessing a hereditary structure. J. Phys. A Math. Gene 1993, 26, L1169-L1174. [CrossRef]

11. Lian, Z.; Lou, S. Symmetries and strong symmetries of the (3+1)-Dimensional Burgers equation. Chin. Phys. Lett. 2004, 21, 219-222.

12. Ting, S. Explicit solutions for a modified 2+1-dimensional coupled Burgers equation by using Darboux transformation. Appl. Math. Lett. 2017, 69, 15-21.

13. Lei, Y.; Yang, D. Finite symmetry transformation group and localized structures of the (2+1)-dimensional coupled Burgers equation. Chin. Phys. B 2013, 22, 040202. [CrossRef]

14. Yucel, C.; Dumitru, B.; Ali, K.; Orkun, T. New exact solutions of Burgers type equations with conformable derivative. Waves Random Complex Media 2017, 27, 103-116.

15. Dumitru, B.; Mustafa, I.; Abdullahi, Y.; Aliyu, I. Lie symmetry analysis and conservation laws for the time fractional simplified modified Kawahara equation. Open Phys. 2018, 16, 302-310.

16. Olver, P.J. Application of Lie Group to Differential Equation; Springer: New York, NY, USA, 1986.

17. Tian, C. Lie Groups and its Applications to Differential Equations; Science Press: Beijing, China, 2001. (In Chinese)

18. Wang, G.W.; Liu, X.Q.; Zhang, Y.Y. Symmetry reduction, exact solutions and conservation laws of a new fifth-order nonlinear integrable equation. Commun. Nonlinear Sci. Numer. Simulat. 2013, 18, 2313-2320. [CrossRef]

19. Lou, S.Y.; Ma, H.C. Non-Lie symmetry groups of (2+1)-dimensional nonlinear systems obtained from a simple direct method. J. Phys. A 2005, 38, L129-L137. [CrossRef]

(C) 2019 by the authors. Licensee MDPI, Basel, Switzerland. This article is an open access article distributed under the terms and conditions of the Creative Commons Attribution (CC BY) license (http:/ / creativecommons.org/licenses/by/4.0/). 